\title{
Highly Selective Hydrodeoxygenation of Dibenzofuran into Bicyclohexane over
}

\section{Hierarchical Pt/ZSM-5 Catalysts}

Xiaoxue $\mathrm{Li}^{\dagger}$, Xiaopo Niuł, Shuaikang Zhu, Shuang Xu, Zheyuan Wang, Xiangwen Zhang, Qingfa Wang*

Key Laboratory for Green Chemical Technology of Ministry of Education, School of Chemical Engineering and Technology, Tianjin University, Tianjin 300072, P. R. China

Collaborative Innovation Center of Chemical Science and Engineering (Tianjin), Tianjin University, Tianjin 300072, P. R. China

$\dagger$ These authors contributed equally to this work and should be regarded as co-first authors

*Corresponding Author: qfwang@tju.edu.cn; Tel: +86-22-27892340
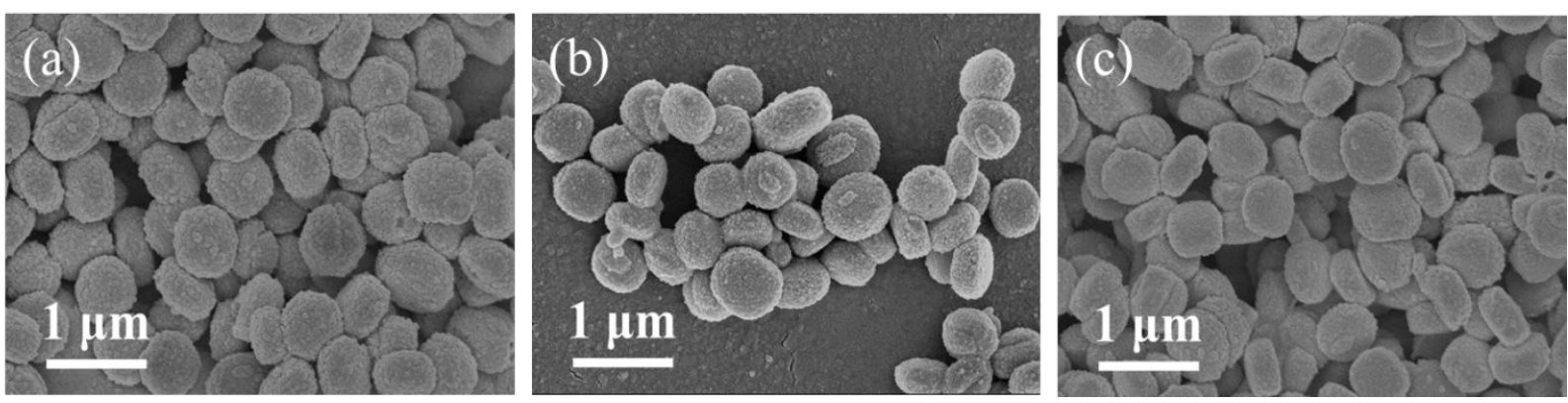

Fig. S1 FE-SEM images of (a) HZ-50, (b) HZ-75 and (c) HZ-100 zeolites. 
Table S1 Pt 4f XPS quantitative results of series catalysts

\begin{tabular}{ccc}
\hline & \multicolumn{2}{c}{ Peak Position } \\
\cline { 2 - 3 } Catalyst & ${\mathrm{Pt} 4 \mathrm{f}_{7 / 2}}$ & ${\mathrm{Pt} 4 \mathrm{f}_{5 / 2}}$ \\
\hline $\mathrm{Pt} / \mathrm{HZ}-50$ & 71.60 & 74.90 \\
$\mathrm{Pt} / \mathrm{HZ}-75$ & 71.91 & 75.21 \\
$\mathrm{Pt} / \mathrm{HZ}-100$ & 71.57 & 74.87 \\
\hline
\end{tabular}
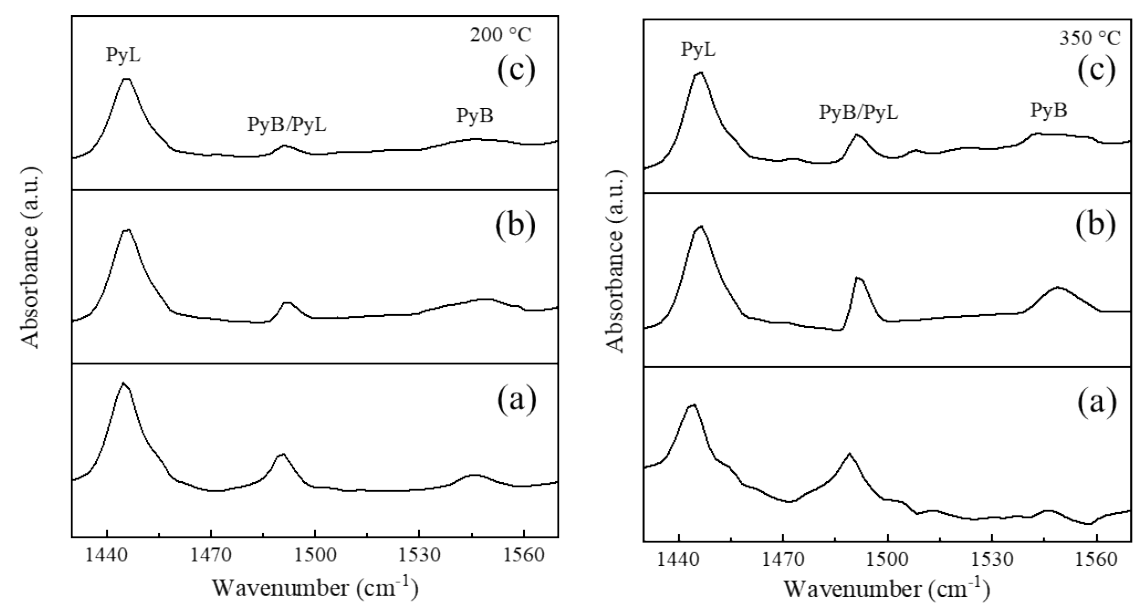

Fig. S2 Py-IR spectra of (a) Pt/HZ-50, (b) Pt/HZ-75 and (c) Pt/HZ-100 catalysts. 
Table S2 Acidity properties of the series Pt/HZ-x catalysts

\begin{tabular}{|c|c|c|c|c|c|c|}
\hline \multirow{2}{*}{ catalysts } & \multicolumn{2}{|c|}{ strong acid $(\mu \mathrm{mol} / \mathrm{g})$} & \multicolumn{2}{|c|}{ weak acid $(\mu \mathrm{mol} / \mathrm{g})$} & \multirow{2}{*}{ total } & \multirow{2}{*}{$\mathrm{B}_{\mathrm{T}} / \mathrm{L}_{\mathrm{T}}$} \\
\hline & B & $\mathrm{L}$ & B & $\mathrm{L}$ & & \\
\hline $\mathrm{Pt} / \mathrm{HZ}-50$ & 16.3 & 53.6 & 13.2 & 65.1 & 148.2 & 0.2485 \\
\hline $\mathrm{Pt} / \mathrm{HZ}-75$ & 9.9 & 46.3 & 5.7 & 53.8 & 115.7 & 0.1558 \\
\hline $\mathrm{Pt} / \mathrm{HZ}-100$ & 4.8 & 39.8 & 3.7 & 44.3 & 92.6 & 0.1011 \\
\hline
\end{tabular}

